\title{
Transcriptomic analysis of COVID-19 lungs and bronchoalveolar lavage fluid samples reveals predominant $B$ cell activation responses to infection
}

\author{
EUGENIO CAVALLI ${ }^{1}$, MARIA CRISTINA PETRALIA ${ }^{1}$, MARIA SOFIA BASILE ${ }^{2}$, \\ ALESSIA BRAMANTI $^{2}$, PLACIDO BRAMANTI ${ }^{2}$, FERDINANDO NICOLETTI ${ }^{1}$, \\ DEMETRIOS A. SPANDIDOS ${ }^{3}$, YEHUDA SHOENFELD ${ }^{4,5}$ and PAOLO FAGONE ${ }^{1}$ \\ ${ }^{1}$ Department of Biomedical and Biotechnological Sciences, University of Catania, I-95123 Catania; \\ '2IRCCS (Scientific Institute for Research, Hospitalization and Healthcare) Centro Neurolesi 'Bonino-Pulejo', \\ I-98124 Messina, Italy; ${ }^{3}$ Laboratory of Clinical Virology, Medical School, University of Crete, \\ 71003 Heraklion, Greece; ${ }^{4}$ Zabludowicz Center for Autoimmune Diseases, Sheba Medical Center \\ (Affiliated to Tel-Aviv University), Tel-Hashomer 5265601, Israel; ${ }^{5}$ I.M. Sechenov \\ First Moscow State Medical University (Sechenov University), 119146 Moscow, Russia
}

Received July 31, 2020; Accepted August 11, 2020

DOI: $10.3892 /$ ijmm.2020.4702

\begin{abstract}
The outbreak of the 2019 coronavirus disease (named, COVID-19), caused by the novel SARS-CoV-2 virus, represents a worldwide severe threat to public health. It is of the utmost importance to characterize the immune responses against the SARS-CoV-2 and the mechanisms of hyperinflammation, in order to design better therapeutic strategies for COVID-19. In the present study, a transcriptomic analysis was performed to profile the immune signatures in lung and the bronchoalveolar lavage fluid samples from COVID-19 patients and controls. Our data concordantly revealed increased humoral responses to infection. The elucidation of the host responses to SARS-CoV-2 infection may further improve our understanding of COVID-19 pathogenesis and suggest better therapeutic strategies.
\end{abstract}

\section{Introduction}

The severe acute respiratory syndrome coronavirus-2 (SARS-CoV-2) was first isolated at the end of 2019 in China (1-5) and, as of August 3rd, 2020, almost 18 million infected patients and 686,703 deaths have been reported globally (WHO Situation Report-196). However, the actual number of the infected subject is under-estimated and, indeed,

Correspondence to: Professor Ferdinando Nicoletti, Department of Biomedical and Biotechnological Sciences, University of Catania, 89 Via Santa Sofia, I-95123 Catania, Italy

E-mail: ferdinic@unict.it

Key words: COVID-19, SARS-CoV-2, immune response, bioinformatics a recent meta-analysis performed on 50,155 patients from 41 studies, showed that the pooled percentage of asymptomatic infection is $15.6 \%$ (6).

Even if SARS-CoV-2 shares similarities with the other coronaviruses, the higher diffusion rate and the possibility to induce fatal complications, such as severe pneumonia, acute respiratory distress syndrome (ARDS), thrombosis, septic shock and organ failure, make this virus a major public health threat (7-10). Development of COVID-19 complications seems to be dependent on a dramatic release of proinflammatory factors, such as interleukin (IL)-1 $\beta$, IL-6, IL-8, tumor necrosis factor- $\alpha$ (TNF- $\alpha)$ and CXC-chemokine ligand 10 (CXCL10) and CC-chemokine ligand 2 (CCL2) in the infected lung tissue and other peripheral organs (2,11-13), which ultimately leads to a reaction known as cytokine release syndrome (CRS). It is likely that CRS promotes a self-sustaining inflammatory process that contributes to the respiratory failure and the systemic manifestations observed in COVID-19 patients (14). A multicenter study of 150 confirmed COVID-19 cases in Wuhan, China, identified as predictors of mortality both elevated ferritin $(15,16)$ and IL-6 levels, which strengthen the hypothesis that fatality events may be due to a virus-driven hyperinflammation $(2,11)$.

The rapid worldwide diffusion of SARS-COV-2 has propelled both basic science and clinical research studies for the elucidation of the pathogenetic mechanisms underlying COVID-19. The emerging observation that a significant percentage proportions of individuals are asymptomatic, not only suggests that SARS-CoV-2 may have a longer incubation period and higher transmission rate, as compared to other coronaviruses, but also advocates potential differences in the host immune responses to this virus. It is therefore, of the utmost importance to characterize the immune responses put against SARS-CoV-2 and the mechanisms of hyperinflammation, in order to design better therapeutic strategies for 
COVID-19. In the present study, we performed a transcriptomic analysis to profile the immune signatures in lung and the bronchoalveolar lavage fluid samples from COVID-19 patients and controls. Our data concordantly revealed increased humoral responses to infection. The elucidation of the host responses to SARS-CoV-2 infection may further improve our understanding of COVID-19 pathogenesis and suggest better therapeutic strategies.

\section{Materials and methods}

Dataset selection. The NCBI Gene Expression Omnibus (GEO) database (http://www.ncbi.nlm.nih.gov/geo/) was interrogated using the terms 'SARS-CoV-2' and 'COVID-19'. The available datasets were shortlisted if: i) they included whole-genome transcriptomic profiling; ii) included human samples; and iii) were not generated on cancer cell lines. Finally, the GSE150316 and the GSE147507 (17) datasets were selected. GSE150316 is a high throughput sequencing dataset of five autopsy samples from patients deceased due to SARS-CoV-2 infection (2-5 technical replicates for each sample were averaged for the downstream analysis) and five negative control samples.

The GSE147507 dataset was generated from three biological replicates of primary human lung epithelium either infected with SARS-CoV-2 (USA-WA1/2020) at a multiplicity of infection (MOI) of 2 , for $24 \mathrm{~h}$, or mock infected. Total mRNA libraries were prepared using tTruSeq Stranded mRNA LP and cDNA libraries sequenced using an Illumina NextSeq 500 platform. Raw reads were aligned to the human genome (hg19) using the RNA-Seq Alignment App on Basespace (Illumina). The submitter-supplied pre-processed and normalized gene expression matrix was used for the analysis.

For the transcriptomic analysis of COVID-19 BALF samples, RNA-Seq data from the Genome Sequence Archive of the Beijing Institute of Genomics (BIG) Data Center (https://bigd.big.ac.cn/) (accession no. CRA002390), and from the NCBI SRA database (accession nos. SRR10571724, SRR10571730 and SRR10571732) (18) were used.

Enrichment and network analysis. Functional enrichment analysis was conducted using the web-based utility, Metascape (19). Metascape analysis makes use of public databanks, such as Gene Ontology, KEGG, and MSigDB, and aggregates enriched ontology terms into non-redundant groups, by calculating the similarity between any two terms (19). Metascape uses the hypergeometric test and the Benjamini-Hochberg p-value correction to identify statistically significant enriched terms. Representative terms from the enrichment analysis are presented as a network. Each term is represented by a node, with its size being proportional to the number of input genes belonging to that term, and the color representing its corresponding cluster. Terms with a similarity score $>0.3$ are linked by an edge. The thicker the edge, the higher the similarity score. The network is visualized using Cytoscape (version 3.1.2) with 'force-directed' layout. One term from each cluster has its description shown as a label.

Computational deconvolution of infiltrating immune cells. In order to evaluate the relative proportions of immune cell subsets in COVID-19 and healthy control samples, we performed a computational deconvolution analysis. To this end, we used the xCell software, a web computational utility that aims at evaluating, by using gene signatures, the relative proportions in a sample of various immune cell types, including immature dendritic cells (iDCs), conventional DCs (cDCs), active DCs (aDCs), plasmacytoid DCs (pDCs), B cells, $\mathrm{CD}^{+}{ }^{+} \mathrm{T}$ cells, memory cells, Th1 cells, Th2 and Treg cells and macrophages (20).

Statistical analysis. The differential expression analysis was performed using the DeSeq2 function. The web-based application NeworkAnalist was used for the statistical analyses. Genes with an adjusted P-value $<0.05$ were identified as differentially expressed genes (DEGs) and selected for further analysis.

Linear regression and Spearman's correlation were performed to compare the expression levels of genes in COVID-19 samples as compared to healthy control samples, the GSE150316 and the GSE147507 datasets.

For the analysis of the deconvolution data, normality was first assessed using the Shapiro-Wilk, D'Agostino-Pearson and Kolmogorov-Smirnov tests. Based on the results, differential analysis as performed using the non-parametric Mann-Whitney U test.

The GraphPad Prism (version 8) software (GraphPad Software, Inc.) and the SPSS software (SPSS, Inc.) were used for the statistical analysis and the generation of the graphs. Unless otherwise stated, $\mathrm{P}<0.05$ was considered to indicate a statistically significant difference.

\section{Results}

Network and enrichment analysis of SARS-CoV-2 infection. In order to determine the transcriptomic signature of lung tissues from COVID-19 patients, we analyzed the GSE150316 RNA-Seq dataset. A total of 55 differentially expressed genes was found, of which 32 were upregulated and 23 downregulated. Gene term enrichment analysis identified GO:0002377: immunoglobulin production, GO:0006959: humoral immune response and GO:0002758: innate immune response-activating signal transduction, as significantly enriched among the upregulated genes (Fig. 1A). A heatmap of the genes belonging to the GO:0002377 (immunoglobulin production) category is presented in Fig. 1B. Among the downregulated genes, GO:0071236: cellular response to antibiotic, GO:0048511: rhythmic process, GO:0042698: ovulation cycle, GO:0019221: cytokine-mediated signaling pathway and GO:0001503: ossification, were found to be significantly enriched (Fig. 1A).

We have previously interrogated the GSE147507 dataset, which included transcriptomic data from primary human bronchial epithelial cells infected in vitro with the SARS-CoV-2 virus (18). Here, we compared this gene signature to the transcriptomic signature of lung biopsies from COVID-19 patients. The publicly available GSE150316 dataset was used in order to perform a correlation analysis on the modulation of the genes perturbed upon SARS-CoV-2 infection and the corresponding genes in GSE147507. A total of 9602 genes were in common between the two datasets. As shown in Fig. 1A, a moderate but significant correlation is found in the transcriptomic profile of 


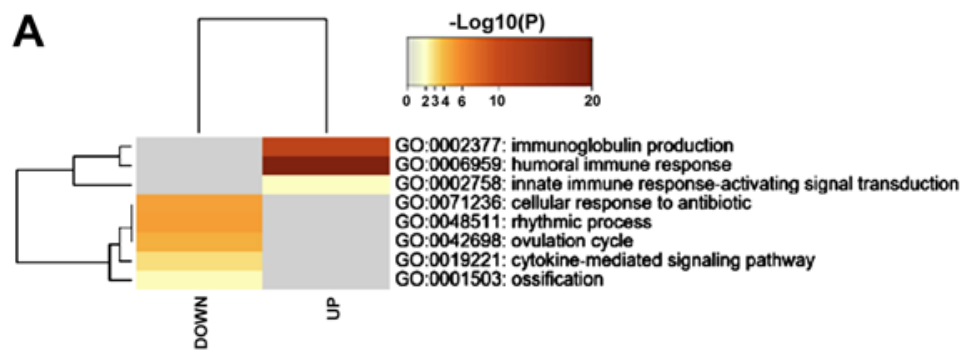

B

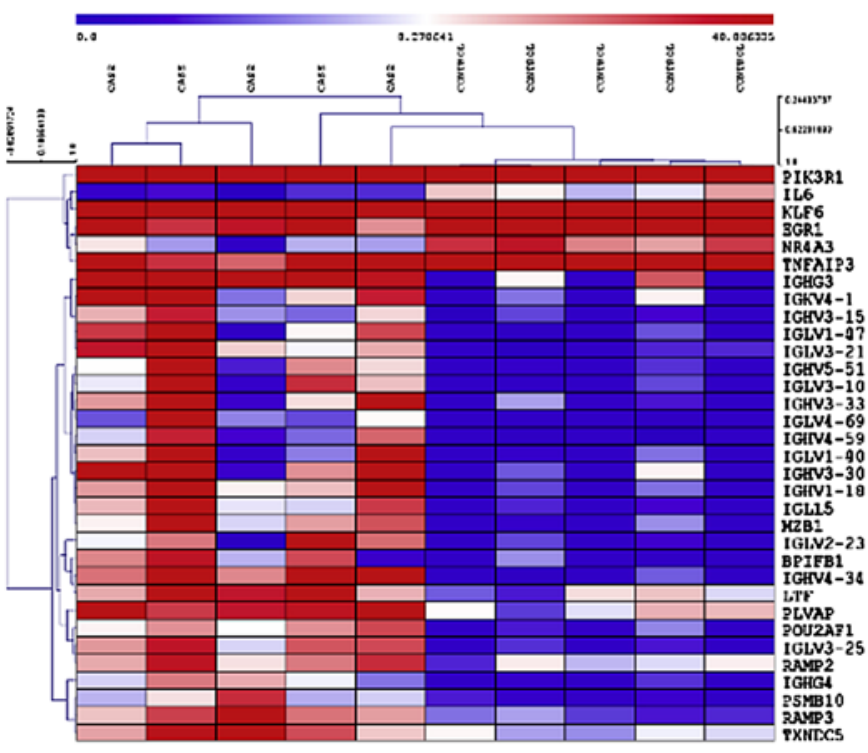

Figure 1. Enrichment and network analysis of COVID-19 lung samples. (A) Hierarchical clustering of the top most enriched terms among the genes significantly upregulated and downregulated in the GSE150316 dataset. The heatmap is colored based on the p-values, and grey cells indicate the lack of significant enrichment. (B) Gene expression heatmap of the genes belonging to the GO:0002377 (immunoglobulin production) category, in lung biopsies of COVID-19 patients and control samples, as determined in the GSE150316 dataset.

A
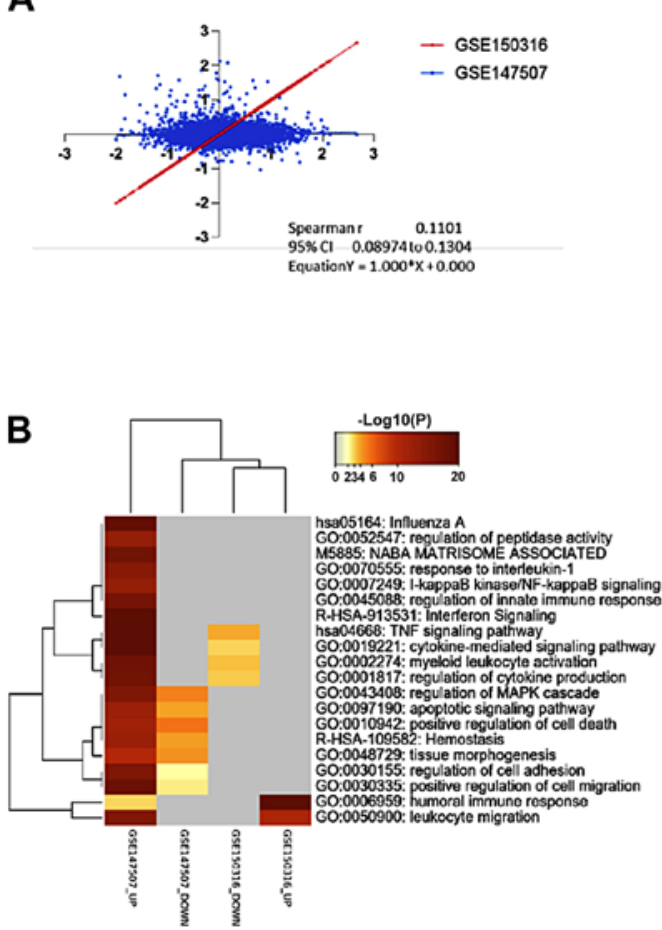

C

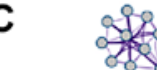

भी8
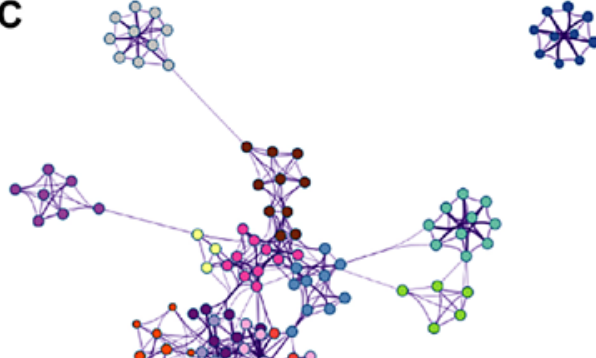
A

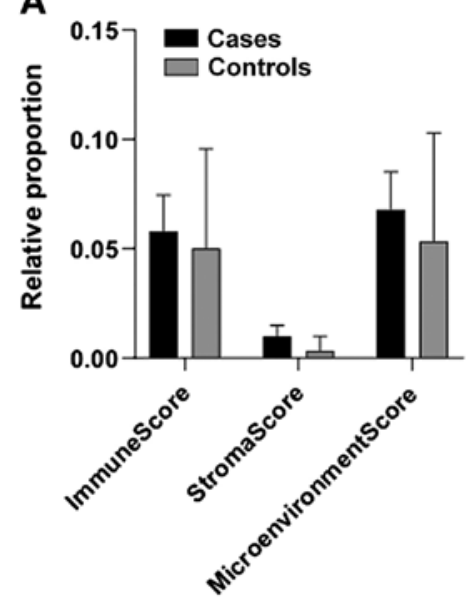

B

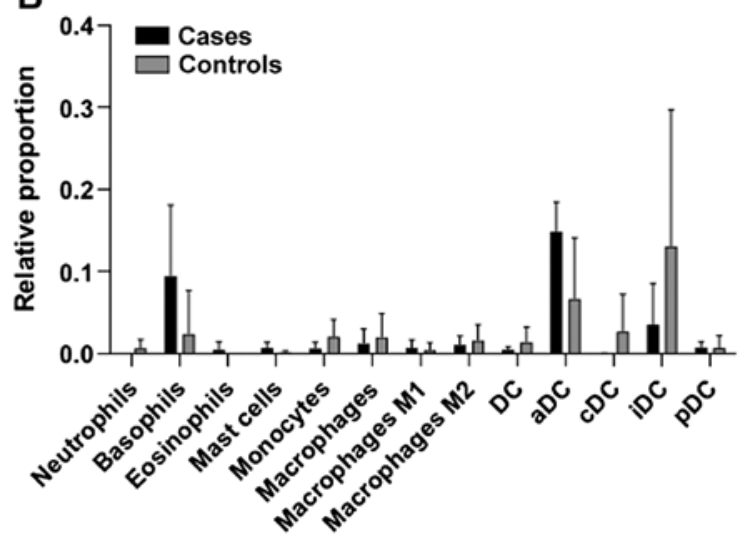

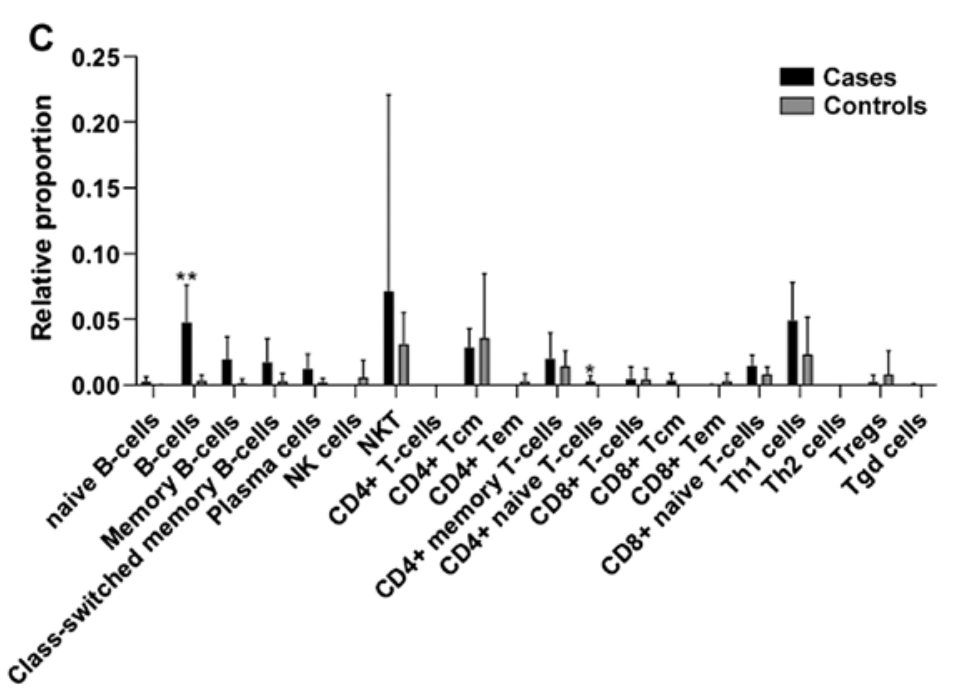

Figure 3. Deconvolution analysis of infiltrating immune cells in lung samples from COVID-19 patients and controls as determined in the GSE150316. (A) Histogram plot for the ImuneScore, StromaScore an MicroenviromentScore in lung samples from COVID-19 patients and controls, as determined in the GSE150316. (B) Relative proportions of infiltrating myeloid cells in lung samples from COVID-19 patients and controls, as determined in the GSE150316. (C) Relative proportions of infiltrating lymphoid cells in lung samples from COVID-19 patients and controls, as determined in the GSE150316.

in vitro infected bronchial epithelial cells and lung samples from patients (Fig. 2A).

Gene term enrichment analysis for the significantly modulated genes identified pathways in common between the GSE147507 and the GSE150316 datasets. The common enriched terms for the upregulated genes in the two datasets were: 'humoral immune response' (GO:0006959) and 'leukocyte migration' (GO:0050900) (Fig. 2B).

Interestingly, the top terms enriched among the downregulated genes in the GSE150316 dataset were: 'TNF signaling pathway' (hsa04668), 'cytokine-mediated signaling pathway' (GO:0019221), 'myeloid leukocyte activation' (GO:0002274) and 'regulation of cytokine production' (GO:0001817) (Fig. 2B). Representative terms from the enrichment analysis and their functional connections are presented as a network (Fig. 2C).

Deconvolution analysis of infiltrating immune cells in lung samples from COVID-19 patients. We next characterized the relative proportions of infiltrating immune cells in the lungs of COVID-19 patients. A shown in Fig. 3A, a moderate, but not significant, increase in the immune score and microenvi- roment score was detected for the COVID-19 lung samples. Also, a moderate, non-significant increase in the percentage of infiltrating basophils and aDCs was observed (Fig. 3B). Analysis of the lymphoid cells in the lungs of COVID-19 patients revealed a significant higher proportion of infiltrating B cells upon SARS-CoV-2 infection, along with a moderate, non-significant increase in NKT and Th1 cells (Fig. 3C).

Characterization of the transcriptomic profile of BALF samples. Next, we compared the gene signature of BALF samples from COVID-19 patients and controls. A total of 3003 genes were found to be modulated in SARS-CoV-2 patients (adjusted $\mathrm{P}$-value $<0.05$ and Ifold-changel $>2$ ), with 1745 genes being upregulated and 1258 genes downregulated. As shown in Fig. 4A, among both the upregulated and downregulated genes, pathways related to cell morphology (GO:0051017; GO:0030155; GO:0030036; GO:0030031) and survival (GO:0010942; GO:0097190) were significantly enriched.

Analysis of the transcription factors identified RELA, NFKB1, USF2 and SP1, as putatively involved in the regulation of the differentially expressed genes (Fig. 4B). 

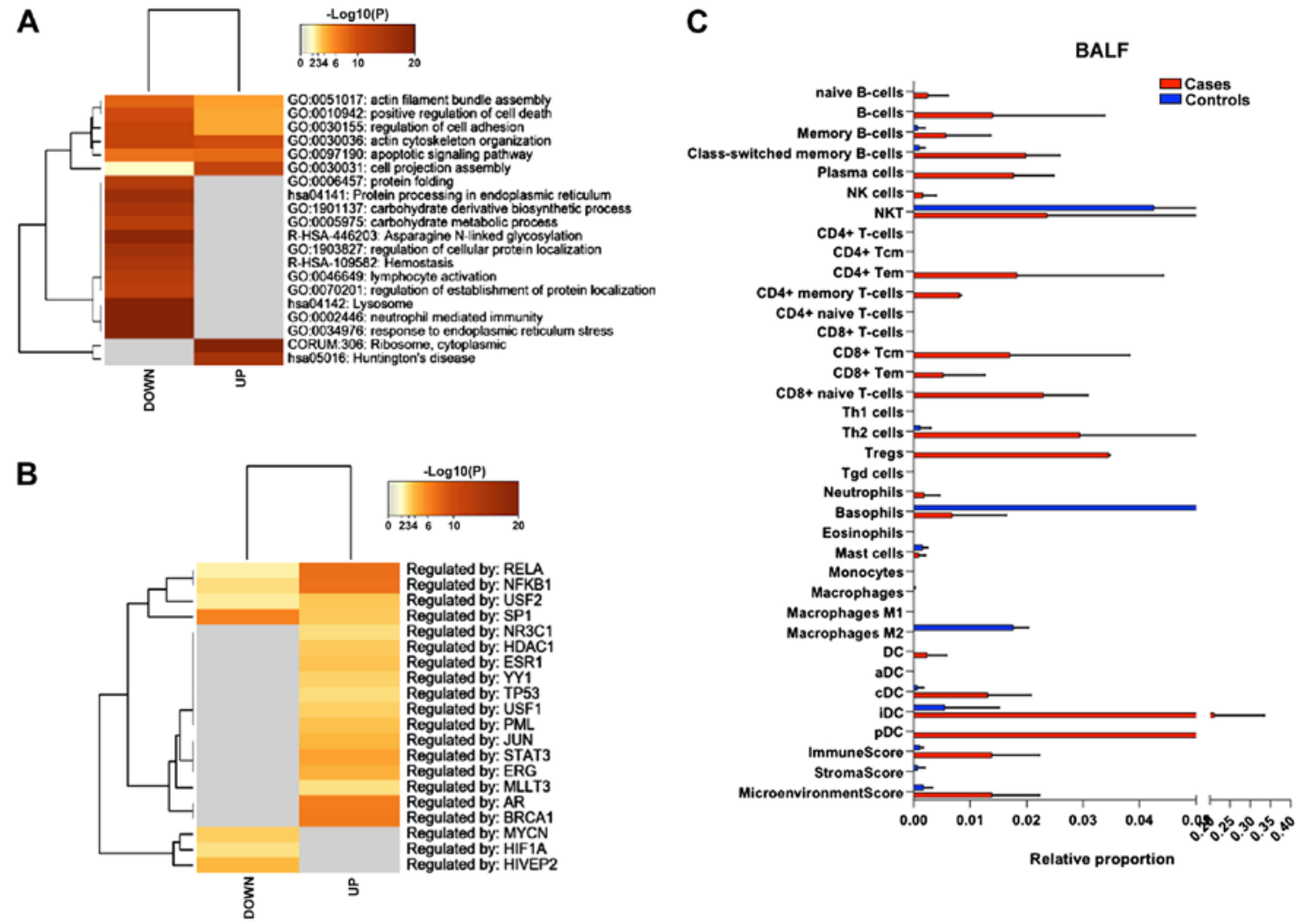

Figure 4. Characterization of the transcriptomic profile of BALF samples from COVID-19 patients and controls. (A) Hierarchical clustering of the top most enriched terms by genes significantly upregulated and downregulated in the BALF samples of COVID-19 patients as compared to control samples. (B) Hierarchical clustering of the top predicted transcription factors involved in the expression of the genes significantly upregulated and downregulated in the BALF samples of COVID-19 patients as compared to control samples. (C) Deconvolution analysis of infiltrating immune cells in BALF samples from COVID-19 patients and controls.

Immune cell deconvolution analysis revealed a trend of higher proportion in B cells (both naïve, memory and plasma cells), along with an increase in CD4 memory T cells, CD8 $\mathrm{T}$ cells and DCs (cDCs, iDCs and pDCs) (Fig. 4C).

\section{Discussion}

The characterization of the exact pathogenetic mechanisms by which SARS-CoV-2 induces multiple organ damage are of immediate importance. Emerging data seem to indicate that beside lungs, other organs, including heart, kidney and the central nervous system may also be affected in COVID-19 (21,22). Patients may show proteinuria, hematuria and increased creatinine levels (21), and may suffer from neurological symptoms, such as headache, epilepsy, disturbed consciousness, anosmia and dysgeusia (22). Some COVID-19 patients also develop thromboembolic events, with elevation of D-dimer and other procoagulant parameters (23), which may represent a secondary anti-phospholipid syndrome (APS) (24), as well as other autoimmune diseases (25). Indeed, accumulating case reports show that COVID-19 patients tested positive for anti-CL, anti-b2-GPI autoantibodies (26-29), as well as lupus anticoagulant $(27,30,31)$.

The use of gene expression profiling data has been extensively employed for the identification of novel pathogenic pathways and therapeutic targets (32-36) for several disorders including, autoimmune diseases (37-40) and cancer $(41,42)$. A computational analysis was performed in order to characterize the immune response to SARS-CoV-2 infection. To achieve this, we exploited publicly available RNA-seq data, generated from lung biopsies and BALF samples from COVID-19 patients. Our data from lung and BALF samples concordantly show that $\mathrm{B}$ cell responses mainly characterize SARS-CoV-2 infection.

It has been already described that SARS-CoV-2 elicits a robust humoral cell response, with virus-specific $\operatorname{IgM}, \operatorname{IgG}$ and $\operatorname{IgA}$, and neutralizing IgG antibodies following infection. Seroconversion usually occurs in most COVID-19 patients between one to two weeks after overt symptoms, and antibody titers last for weeks, following virus eradication (43). It seems also that protective B cell memory arises following infection, as a recent study of SARS-CoV-2 infection in rhesus macaques found that animals that had resolved the primary infection were resistant to reinfection one month later (44). Also, independent data show that higher virus-specific antibody titers correlated with greater virus neutralization and are inversely correlated with viral load (43). However, higher titers may be associated with more severe clinical cases (45-47), suggesting that the humoral responses may not be sufficient to protect from severe disease. Up to now, there is no evidence that SARS-CoV-2-induced antibodies contribute to some of the pathological features observed in COVID-19 patients. However, this possibility should be taken into consideration in light of the above-mentioned data on secondary APS syndrome in some COVID-19 cases. It has been proposed that antibody-dependent enhancement (ADE), may represent, at least one, of the causes of the CRS (48-51). When the virus infects the body, memory B cells are activated 
while the activation of naive B cells is inhibited. However, both virus-specific antibodies and antibodies cross-reacting with other similar virus strains are produced and secreted. These cross-reactive antibodies may elicit the entry of viruses into macrophages in a $\mathrm{Fc}$ receptor-mediated manner, and consequently, viruses undergo rapid replication and release, resulting in immune dysregulation, and severe illness in patients with COVID-19 (52). The potential role of SARS-CoV-2-induced IgG antibodies in promoting neuroinflammation in SARS-CoV-2 infection should also be mentioned, as ADE occurrence may involve microglia cells following the binding of $\mathrm{Abs}$ to $\mathrm{Fc}$ receptors expressed on these cells.

As the mTOR pathway plays a fundamental role in B-cell development via the control of BCL6 expression in B cells from the germinal center (53), it is reasonable to believe that the use of inhibitors of mTOR, i.e., rapamycin and 'rapalogs', could reduce the populations of antigen-specific memory $\mathrm{B}$ cells and limit the occurrence of ADE in SARS-CoV-2 infected patients. This further strengthen the rational for using mTOR inhibitors in COVID-19, as previously discussed (20). Indeed, by using an anti-signature computational approach, our analysis showed that the mTOR inhibitor, sirolimus, may be a candidate drug to be used in COVID-19 patients, which is in line with data on the activation of the phosphoinositol 3-kinase (PI3K)/AKT/mTOR pathway in response to the infection with another coronavirus, MERS-CoV (54). Also, mTOR has been recognized as a key factor in regulating the replication of viruses $(36,54-57)$, and in patients with H1N1 pneumonia, early treatment with corticosteroids in combination with rapamycin has been associated with improvement in multiple organ dysfunction, virus clearance, and shortened time in ventilators (58).

Hence, the use of mTOR inhibitors may have many-fold advantages on the course od SARS-CoV-2 infection, which could improve lung pathology, but also, the peripheral manifestations of the disease, including the CNS.

Interestingly, our data suggest potential reasons for the gender differences in COVID-19 susceptibility (2). Indeed, the prevalence in men is between 55 and 68\% (59) and increased clinical severity and mortality has been reported (60). Certainly, female-specific hormonal factors can be involved. In this regard, it is notable that among the upregulated genes in BALF from COVID-19 patients, $22(\log (\mathrm{q})$ value $=-4)$ are regulated by AR (androgen receptor), while 15 are regulated by ESR 1 (estrogen receptor 1$)(\log (\mathrm{q})$ value $=-1.8)$. AR is known to play a key role in both innate and adaptive immune responses $(61,62)$, and ESR1 has been recognized as a regulator of interferon production and anti-viral responses (63). These observations may underly the different clinical response to SAR-CoV-2 infection in women and men. It is important to note that selective estrogen receptor modulators. such as toremifene, have already been proposed as potential drugs to treat coronavirus infections (64). These observations point to biological processes that may explain the lower female incidence and lethality of SARS-CoV-2 infection, offering candidate therapeutic options in patients suffering from COVID-19.

Finally, we have to acknowledge some of the limitations of the present study. First, the differentially expressed genes, that we have prioritized in our study, and the deconvolution analysis have been obtained from a really small cohort of patients, hence the data may be biased, due to the high degree of interindividual variability that characterize SARS-CoV-2 infection. Lung-specific gene expression profiles from homogeneous COVID-19 patients will allow to better identify prognostic predictors and tailored therapeutic strategies. Second, the deconvolution analysis of the immune populations does not allow to assess the functionality of the immune cells and their actual involvement in COVID-19 pathology.

\section{Acknowledgements}

Not applicable.

\section{Funding}

This study was supported by current research funds 2020 of IRCCS Centro Neurolesi ‘Bonino-Pulejo', Messina, Italy.

\section{Availability of data and materials}

The datasets analyzed during the current study are available on the NCBI Gene Expression Omnibus (GEO) database (http://www.ncbi.nlm.nih.gov/geo/) under the accession nos. GSE150316 and GSE147507, on the Genome Sequence Archive of the Beijing Institute of Genomics (BIG) Data Center (https://bigd.big.ac.cn/) (accession no. CRA002390) and on the NCBI SRA database (accession nos. SRR10571724, SRR10571730 and SRR10571732).

\section{Authors' contributions}

Conceptualization: DAS, YS, FN and PF; data curation: $\mathrm{EC}, \mathrm{MCP}$ and PF; formal analysis: $\mathrm{EC}, \mathrm{MCP}, \mathrm{MSB}$ and $\mathrm{AB}$; funding acquisition: $\mathrm{PB}$; methodology: $\mathrm{PF}$; writing original draft: EC, AB; review and editing: PB, DAS, YS, FN and PF. All authors read and approved the final manuscript.

\section{Ethics approval and consent to participate}

Not applicable.

\section{Patient consent for publication}

Not applicable.

\section{Competing interests}

DAS is the Editor-in-Chief for the journal, but had no personal involvement in the reviewing process, or any influence in terms of adjudicating on the final decision, for this article. The other authors declare that they have no competing interests.

\section{References}

1. Zhu N, Zhang D, Wang W, Li X, Yang B, Song J, Zhao X, Huang B, Shi W, Lu R, et al; China Novel Coronavirus Investigating and Research Team: A Novel Coronavirus from Patients with Pneumonia in China, 2019. N Engl J Med 382: 727-733, 2020.

2. Xu J, Zhao S, Teng T, Abdalla AE, Zhu W, Xie L, Wang Y and Guo X: Systematic comparison of two animal-to-human transmitted human Coronaviruses: SARS-CoV-2 and SARS-CoV. Viruses 12: 12, 2020. 
3. Cucinotta D and Vanelli M: WHO Declares COVID-19 a Pandemic. Acta Biomed 91: 157-160, 2020.

4. Chen L, Jin Q, Zhou Y, Yang J, Wang Z, Ge K, Yang J and Wang H: Clinical characteristics of 2019 novel coronavirus pneumonia in Zhejiang province, China. Mol Med Rep 22: 2583-2587, 2020.

5. Kanduc D and Shoenfeld Y: On the molecular determinants of the SARS-CoV-2 attack. Clin Immunol 215: 108426, 2020.

6. He J, Guo Y, Mao R and Zhang J: Proportion of asymptomatic coronavirus disease 2019 (COVID-19): A systematic review and meta-analysis. J Med Virol jmv.26326, 2020.

7. Chen N, Zhou M, Dong X, Qu J, Gong F, Han Y, Qiu Y, Wang J, Liu Y, Wei Y, et al: Epidemiological and clinical characteristics of 99 cases of 2019 novel coronavirus pneumonia in Wuhan, China: A descriptive study. Lancet 395: 507-513, 2020.

8. Docea AO, Tsatsakis A, Albulescu D, Cristea O, Zlatian O, Vinceti M, Moschos SA, Tsoukalas D, Goumenou M, Drakoulis N, et al: A new threat from an old enemy: Re-emergence of coronavirus (Review). Int J Mol Med 45: 1631-1643, 2020.

9. Kostoff RN, Briggs MB, Porter AL, Aschner M, Spandidos DA and Tsatsakis A: [Editorial] COVID-19: Post-lockdown guidelines. Int J Mol Med 46: 463-466, 2020.

10. Shoenfeld Y: Corona (COVID-19) time musings: Our involvement in COVID-19 pathogenesis, diagnosis, treatment and vaccine planning. Autoimmun Rev 19: 102538, 2020.

11. Mehta P, McAuley DF, Brown M, Sanchez E, Tattersall RS and Manson JJ; HLH Across Speciality Collaboration, UK COVID-19: Consider cytokine storm syndromes and immunosuppression. Lancet 395: 1033-1034, 2020.

12. Stancioiu F, Papadakis GZ, Kteniadakis S, Izotov BN, Coleman MD, Spandidos DA and Tsatsakis A: A dissection of SARS-CoV-2 with clinical implications (Review). Int J Mol Med 46: 489-508, 2020.

13. Kerslake R, Hall M, Randeva HS, Spandidos DA, Chatha K, Kyrou I and Karteris E: Co-expression of peripheral olfactory receptors with SARS-CoV-2 infection mediators: Potential implications beyond loss of smell as a COVID-19 symptom. Int J Mol Med 46: 949-956, 2020

14. Tang N,Li D, Wang X and Sun Z: Abnormal coagulation parameters are associated with poor prognosis in patients with novel coronavirus pneumonia. J Thromb Haemost 18: 844-847, 2020.

15. Ruscitti $P$, Berardicurti $O$, Di Benedetto $P$, Cipriani $P$, Iagnocco $A$, Shoenfeld Y and Giacomelli R: Severe COVID-19, Another Piece in the Puzzle of the Hyperferritinemic Syndrome. An Immunomodulatory Perspective to Alleviate the Storm. Front Immunol 11: 1130, 2020.

16. Perricone C, Bartoloni E, Bursi R, Cafaro G, Guidelli GM, Shoenfeld Y and Gerli R: COVID-19 as part of the hyperferritinemic syndromes: The role of iron depletion therapy. Immunol Res 68: 213-224, 2020.

17. Blanco-Melo D, Nilsson-Payant BE, Liu WC, Møller R, Panis M, Sachs D, Albrecht RA and tenOever BR: SARS-CoV-2 launches a unique transcriptional signature from in vitro, ex vivo, and in vivo systems. bioRxiv: doi: https://doi.org/10.1101/2020.03.24.004655.

18. Fagone P, Ciurleo R, Lombardo SD, Iacobello C, Palermo CI, Shoenfeld $\mathrm{Y}$, Bendtzen $\mathrm{K}$, Bramanti $\mathrm{P}$ and Nicoletti $\mathrm{F}$ : Transcriptional landscape of SARS-CoV-2 infection dismantles pathogenic pathways activated by the virus, proposes unique sex-specific differences and predicts tailored therapeutic strategies. Autoimmun Rev 19: 102571, 2020.

19. Zhou Y, Zhou B, Pache L, Chang M, Khodabakhshi AH, Tanaseichuk O, Benner C and Chanda SK: Metascape provides a biologist-oriented resource for the analysis of systems-level datasets. Nat Commun 10: 1523, 2019.

20. Aran D, Hu Z and Butte AJ: xCell: Digitally portraying the tissue cellular heterogeneity landscape. Genome Biol 18: 220, 2017.

21. Cheng Y, Luo R, Wang K, Zhang M, Wang Z, Dong L, Li J, Yao Y, $\mathrm{Ge} S$ and $\mathrm{Xu} \mathrm{G}$ : Kidney disease is associated with in-hospital death of patients with COVID-19. Kidney Int 97: 829-838, 2020.

22. Wu Y, Xu X, Chen Z, Duan J, Hashimoto K, Yang L, Liu C and Yang C: Nervous system involvement after infection with COVID-19 and other coronaviruses. Brain Behav Immun 87: $18-22,2020$.

23. Han H, Yang L, Liu R, Liu F, Wu KL, Li J, Liu XH and Zhu CL: Prominent changes in blood coagulation of patients with SARS-CoV-2 infection. Clin Chem Lab Med 58: 1116-1120 2020

24. Cavalli E, Bramanti A, Ciurleo R, Tchorbanov AI, Giordano A, Fagone P, Belizna C, Bramanti P, Shoenfeld Y and Nicoletti F: Entangling COVID-19 associated thrombosis into a secondary antiphospholipid antibody syndrome: Diagnostic and therapeutic perspectives (Review). Int J Mol Med 46: 903-912, 2020.
25. Ehrenfeld M, Tincani A, Andreoli L, Cattalini M, Greenbaum A, Kanduc D, Alijotas-Reig J, Zinserling V, Semenova N, Amital H, et al: Covid-19 and autoimmunity. Autoimmun Rev 19: 102597, 2020

26. Zhang Y, Xiao M, Zhang S, Xia P, Cao W, Jiang W, Chen H, Ding $\mathrm{X}$, Zhao $\mathrm{H}$, Zhang $\mathrm{H}$, et al: Coagulopathy and antiphospholipid antibodies in patients with Covid-19. N Engl J Med 382: e38, 2020

27. Beyrouti R, Adams ME, Benjamin L, Cohen H, Farmer SF, Goh YY, Humphries F, Jäger HR, Losseff NA, Perry RJ, et al: Characteristics of ischaemic stroke associated with COVID-19. J Neurol Neurosurg Psychiatry 91: 889-891, 2020.

28. Harzallah I, Debliquis A and Drenou B: Lupus anticoagulant is frequent in patients with Covid-19. J Thromb Haemost 18: 2064-2065, 2020.

29. Zayet S, Klopfenstein T, Kovacs R, Stancescu S and Hagenkötter B: Acute cerebral stroke with multiple infarctions and COVID-19, France, 2020. Emerg Infect Dis 26: 26, 2020.

30. Helms J, Tacquard C, Severac F, Leonard-Lorant I, Ohana M, Delabranche X, Merdji H, Clere-Jehl R, Schenck M, Fagot Gandet F, et al; CRICS TRIGGERSEP Group (Clinical Research in Intensive Care and Sepsis Trial Group for Global Evaluation and Research in Sepsis): High risk of thrombosis in patients with severe SARS-CoV-2 infection: A multicenter prospective cohort study. Intensive Care Med 46: 1089-1098, 2020.

31. Sieiro Santos C, Nogal Arias C, Moriano Morales C, Ballesteros Pomar M, Diez Alvarez E and Perez Sandoval T: Antiphospholipid antibodies in patient with acute lower member ischemia and pulmonary thromboembolism as a result of infection by SARS-CoV2. Clin Rheumatol 39: 2105-2106, 2020.

32. Fagone P, Mangano K, Quattrocchi C, Motterlini R, Di Marco R, Magro G, Penacho N, Romao CC and Nicoletti F: Prevention of clinical and histological signs of proteolipid protein (PLP)-induced experimental allergic encephalomyelitis (EAE) in mice by the water-soluble carbon monoxide-releasing molecule (CORM)-A1. Clin Exp Immunol 163: 368-374, 2011.

33. Fagone P, Mangano K, Coco M, Perciavalle V, Garotta G, Romao CC and Nicoletti F: Therapeutic potential of carbon monoxide in multiple sclerosis. Clin Exp Immunol 167: 179-187, 2012.

34. Cavalli E, Mazzon E, Basile MS, Mangano K, Di Marco R, Bramanti P, Nicoletti F, Fagone P and Petralia MC: Upregulated expression of macrophage migration inhibitory factor, its analogue D-Dopachrome Tautomerase, and the CD44 receptor in peripheral CD4 T cells from clinically isolated syndrome patients with rapid conversion to clinical defined multiple sclerosis. Medicina (Kaunas) 55: 667, 2019.

35. Rothweiler F, Michaelis M, Brauer P, Otte J, Weber K, Fehse B, Doerr HW, Wiese M, Kreuter J, Al-Abed Y, et al: Anticancer effects of the nitric oxide-modified saquinavir derivative saquinavir-NO against multidrug-resistant cancer cells. Neoplasia 12: 1023-1030, 2010.

36. Nicoletti F, Fagone P, Meroni P, McCubrey J and Bendtzen K: mTOR as a multifunctional therapeutic target in HIV infection. Drug Discov Today 16: 715-721, 2011.

37. Lombardo SD, Mazzon E, Basile MS, Cavalli E, Bramanti P, Nania R, Fagone P, Nicoletti F and Petralia MC: Upregulation of IL-1 receptor antagonist in a mouse model of migraine. Brain Sci 9: 172, 2019.

38. Petralia MC, Mazzon E, Fagone P, Falzone L, Bramanti P, Nicoletti F and Basile MS: Retrospective follow-up analysis of the transcriptomic patterns of cytokines, cytokine receptors and chemokines at preconception and during pregnancy, in women with post-partum depression. Exp Ther Med 18: 2055-2062, 2019.

39. Lombardo SD, Mazzon E, Mangano K, Basile MS, Cavalli E, Mammana S, Fagone P, Nicoletti F and Petralia MC: Transcriptomic analysis reveals involvement of the macrophage migration inhibitory factor gene network in Duchenne Muscular Dystrophy. Genes (Basel) 10: 939, 2019.

40. Kosiewicz MM, Auci DL, Fagone P, Mangano K, Caponnetto S, Tucker CF, Azeem N, White SK, Frincke JM, Reading CL, et al: HE3286, an orally bioavailable synthetic analogue of an active DHEA metabolite suppresses spontaneous autoimmune diabetes in the non-obese diabetic (NOD) mouse. Eur J Pharmacol 658: 257-262, 2011.

41. Lombardo SD, Presti M, Mangano K, Petralia MC, Basile MS, Libra M, Candido S, Fagone P, Mazzon E, Nicoletti F, et al: Prediction of PD-L1 expression in neuroblastoma via computational modeling. Brain Sci 9: 221, 2019. 
42. Basile MS, Mazzon E, Russo A, Mammana S, Longo A Bonfiglio V, Fallico M, Caltabiano R, Fagone P, Nicoletti F, et al: Differential modulation and prognostic values of immune-escape genes in uveal melanoma. PLoS One 14: e0210276, 2019.

43. Vabret N, Britton GJ, Gruber C, Hegde S, Kim J, Kuksin M, Levantovsky R, Malle L, Moreira A, Park MD, et al; Sinai Immunology Review Project: Immunology of COVID-19: Current State of the Science. Immunity 52: 910-941, 2020.

44. Bao L, Deng W, Gao H, Xiao C, Liu J, Xue J, Lv Q, Liu J, $\mathrm{Yu} \mathrm{P}, \mathrm{Xu} \mathrm{Y}$, et al: Reinfection could not occur in SARS-CoV-2 infected rhesus macaques. bioRxiv: doi: https://doi. org/10.1101/2020.03.13.990226.

45. Okba NMA, Müller MA, Li W, Wang C, Geurts van Kessel CH, Corman VM, Lamers MM, Sikkema RS, de Bruin E, Chandler FD, et al: Severe acute respiratory syndrome coronavirus 2-specific antibody responses in coronavirus disease patients. Emerg Infect Dis 26: 1478-1488, 2020.

46. Zhao J, Yuan Q, Wang H, Liu W, Liao X, Su Y, Wang X, Yuan J, $\mathrm{Li} \mathrm{T}$, Li J, et al: Antibody responses to SARS-CoV-2 in patients of novel coronavirus disease 2019. Clin Infect Dis: March 28 2020 (Epub ahead of print).

47. Zhou F, Yu T, Du R, Fan G, Liu Y, Liu Z, Xiang J, Wang Y, Song B, Gu X, et al: Clinical course and risk factors for mortality of adult inpatients with COVID-19 in Wuhan, China: A retrospective cohort study. Lancet 395: 1054-1062, 2020.

48. Tetro JA: Is COVID-19 receiving ADE from other coronaviruses? Microbes Infect 22: 72-73, 2020.

49. Cao X: COVID-19: Immunopathology and its implications for therapy. Nat Rev Immunol 20: 269-270, 2020.

50. Iwasaki A and Yang Y: The potential danger of suboptimal antibody responses in COVID-19. Nat Rev Immunol 20: 339-341, 2020.

51. Huang A, Garcia-Carreras B, Hitchings M, Yang B, Katzelnick LC, Rattigan SM, Borgert BA, Moreno CA, Solomon BD, Rodriguez-Barraquer I, et al: A systematic review of antibody mediated immunity to coronaviruses: antibody kinetics, correlates of protection, and association of antibody responses with severity of disease. medRxiv: doi: 10.1101/2020.04.14.20065771

52. Zheng Y, Li R and Liu S: Immunoregulation with mTOR inhibitors to prevent COVID-19 severity: A novel intervention strategy beyond vaccines and specific antiviral medicines. J Med Virol: May 22, 2020 (Epub ahead of print).

53. Raybuck AL, Cho SH, Li J, Rogers MC, Lee K, Williams CL, Shlomchik M, Thomas JW, Chen J, Williams JV, et al: B cell-intrinsic mTORC1 promotes germinal center-defining transcription factor gene expression, somatic hypermutation, and memory B cell generation in humoral immunity. J Immunol 200: $2627-2639,2018$
54. Kindrachuk J, Ork B, Hart BJ, Mazur S, Holbrook MR, Frieman MB, Traynor D, Johnson RF, Dyall J, Kuhn JH, et al: Antiviral potential of ERK/MAPK and PI3K/AKT/mTOR signaling modulation for Middle East respiratory syndrome coronavirus infection as identified by temporal kinome analysis. Antimicrob Agents Chemother 59: 1088-1099, 2015.

55. Kuss-Duerkop SK, Wang J, Mena I, White K, Metreveli G, Sakthivel R, Mata MA, Muñoz-Moreno R, Chen X, Krammer F, et al: Influenza virus differentially activates mTORC1 and mTORC2 signaling to maximize late stage replication. PLoS Pathog 13: e1006635, 2017.

56. Nicoletti F, Lapenta C, Donati S, Spada M, Ranazzi A, Cacopardo B, Mangano K, Belardelli F, Perno C and Aquaro S: Inhibition of human immunodeficiency virus (HIV-1) infection in human peripheral blood leucocytes - SCID reconstituted mice by rapamycin. Clin Exp Immunol 155: 28-34, 2009.

57. Donia M, McCubrey JA, Bendtzen K and Nicoletti F: Potential use of rapamycin in HIV infection. Br J Clin Pharmacol 70: 784-793, 2010.

58. Wang CH, Chung FT, Lin SM, Huang SY, Chou CL, Lee KY, Lin TY and Kuo HP: Adjuvant treatment with a mammalian target of rapamycin inhibitor, sirolimus, and steroids improves outcomes in patients with severe H1N1 pneumonia and acute respiratory failure. Crit Care Med 42: 313-321, 2014.

59. Cai H: Sex difference and smoking predisposition in patients with COVID-19. Lancet Respir Med 8: e20, 2020.

60. Li L, Huang T, Wang Y, Wang ZP, Liang Y, Huang TB, Zhang HY, Sun W and Wang Y: COVID-19 patients' clinical characteristics, discharge rate, and fatality rate of meta-analysis. J Med Virol 92: $577-583,2020$.

61. Lai JJ, Lai KP, Zeng W, Chuang KH, Altuwaijri S and Chang C: Androgen receptor influences on body defense system via modulation of innate and adaptive immune systems: Lessons from conditional AR knockout mice. Am J Pathol 181: 1504-1512, 2012.

62. Gubbels Bupp MR and Jorgensen TN: Androgen-induced immunosuppression. Front Immunol 9: 794, 2018.

63. Kovats S: Estrogen receptors regulate innate immune cells and signaling pathways. Cell Immunol 294: 63-69, 2015.

64. Zhou Y, Hou Y, Shen J, Huang Y, Martin W and Cheng F: Network-based drug repurposing for novel coronavirus 2019-nCoV/SARS-CoV-2. Cell Discov 6: 14, 2020.

This work is licensed under a Creative Commons Attribution-NonCommercial-NoDerivatives 4.0 International (CC BY-NC-ND 4.0) License. 